\title{
Drei Kapitel über die Freiheit
}

\section{Wolfgang Kersting (Kiel)}

Drei Kapitel über die Freiheit, find die im Fortgang den Kreis immer enger ziehen. Das erste heisst: Über einige anthropologische Voraussetzungen der Freiheit und ist sehr philosophisch. Das zweite heisst: Über die Zumutungen der Freiheit in der Moderne, die immer grösser werden, je moderner die Moderne wird und ist auch noch philosophisch, aber auch soziologisch. Das dritte heisst: Über die Selbstabschaffung des Bürgers im Sozialstaat und ist sehr politisch, aber auch ein wenig moralisch. Und dann gibt es noch einen Anhang über falsche Toleranz, falsche Neutralität und die Schwierigkeiten liberaler Selbstbehauptung.

\section{1. Über einige anthropologische Voraussetzungen der Freiheit}

Tugenden sind für Nietzsche der subjektive Ausdruck der „Sittlichkeit der Sitte”, das verinnerlichte Abbild petrifizierter Denkund Lebensverhältnisse. Tugend und Sittlichkeit sind Beharrungskräfte, die den Menschen hindern, neue Erfahrungen zu machen, Revisionen vorzunehmen, Beweglichkeit zu gewinnen. Beide stellen sich auch aller Individualisierung, aller existentiellen Selbstfindung entgegen; sie sorgen dafür, dass „die Originalität jeder Art ein böses Gewissen” (Nietzsche, 1967a, S. 20) bekommt, denn der Mensch wird durch die tugendethische Formierung nicht „als Einzelner erzogen, sondern als Glied eines Ganzen, als Ziffer einer Majorität” (Nietzsche, 1967b, S. 48). Er gerät unter das Diktat der „Gemeindegewohnheiten” und kann sich nicht aus der „socialen Zwangsjacke” (Nietzsche, 1967c, S. 309) befreien. Dabei ist die Zeit reif für die grosse Freiheit. „Je weniger die Menschen durch das Herkommen gebunden sind, um so größer wird die innere Bewegung der Motive, um so grösser wiederum, dementsprechend, die äußere Unruhe, das Durcheinanderfluten der Menschen, die Polyphonie der Bestrebungen. Für wen gibt es jetzt noch einen strengen Zwang, an einen Ort sich und seine Nachkommen anzubinden? Für wen gibt es überhaupt noch etwas streng Bindendes? Wie alle Stilarten der Künste nebeneinander nachgebildet werden, so auch alle Stufen und Arten der Moralität, der Sitten, der Kulturen. - Ein solches Zeitalter bekommt seine Bedeutung dadurch, dass in ihm die verschiedenen Weltbetrachtungen, Sitten, Kulturen verglichen und nebeneinander durchlebt werden können” (Nietzsche, 1980, S. 464).

Was bei Nietzsche noch altmodisch Sittlichkeit, Sitte und Tugend hiess, heisst dann bei Heidegger „Man”. Diejenigen, die es vorziehen, sich leben zu lassen, anstatt ihr Leben in die eigenen Hände zu nehmen, unterwerfen sich dem ?Man?. Wir kennen alle dieses Man; es ist eine ebenso gesichtslose wie mächtige Autorität, die unser Denken und Handeln bestimmt, wir tun das, was man tut, wir sehen die Dinge so, wie man sie sieht. Ein Leben unter der Herrschaft des Man, wo alle gleich sind und wir genauso wie die Anderen, nennt Heidegger ein ?uneigentliches? Leben. Es ist ein Leben ohne Eigenbeteiligung, wir sind in einem solchen Leben noch nicht wir selbst, sondern nur eine Filiale des Man. Wir sind noch nicht zu uns selbst gekommen; unser Lebenstext ist noch auf der Suche nach seinem authentischen Autor. Um ein eigentliches Leben zu gewinnen, müssen wir, wie Heidegger sagt, Selbstsein entwickeln, zu einem Selbst werden. Und zu einem Selbst wird man, wenn man sich zu sich selbst entschliesst, wenn man zu sich eine Beziehung der Wertschätzung entwickelt, wenn man sich aus der Vormundschaft des Man befreut und die Sorge um sich selbst zum Mittelpunkt seines Lebens macht.

Dass sich der Freiheitswille nicht damit begnügen kann, den äußeren Zwang abzuschütteln, sondern sich zusätzlich daran machen muss, die vielfältigen inneren Handlungseinschränkungen zu bekämpfen, das Herkommen, die fraglosen Üblichkeiten, die Ideologien und Moden ist für das neuzeitliche Individuum ausgemachte Sache. Die äussere Befreiung muss durch eine innere Befreiung vervollständigt werden. Denn dort, wo wir Eigenes vermuten, wo wir meinen, bei uns selbst zu sein, macht sich in Wirklichkeit eine Besatzungsmacht breit. Um frei zu werden, um unser eigenes Leben zu führen, müssen wir uns von dieser Besatzungsmacht befreien. Wir müssen die fremden Imprägnierungen aus dem Gewebe unseres Inneren entfernen. Erst nachdem wir uns derart von fremden Einflüssen gereinigt haben, können wir uns daran machen, die Herrschaft über unser Leben zu gewinnen, uns selbst zu bestimmen.

Angesichts der neuzeittypischen Selbstbestimmungsemphase mag manchem das schöne Bonmot in den Sinn kommen, dass ein Optimist nur ein schlecht informierter Pessimist ist. Verdankt sich der Selbstbestimmungsoptimismus des modernen Individualismus nicht dem Umstand, dass Wichtiges unterschlagen wird? Ist er nicht Ausdruck einer Hybris, die wieder hermeneutische Demut lernen muss? Menschen sind endlich; ihr Leben ist von Unveränderlichkeiten geradezu umzingelt. Ein Selbstbestimmungskonzept, das den revolutionären Traum von dem absoluten Neubeginn träumt, das die Schöpfung der Welt als Selbstschöpfung wiederholen will, sich aus allen vorfindlichen Wirklichkeiten ins Reich unverbindlicher Potentialitäten begeben möchte, um dann seine eigene Wirklichkeit authentisch aus sich selbst herauszusetzen, ist angesichts der Endlichkeit menschlicher Existenz illusionär. Wir sind Hypoleptiker; uns mit dem abzufinden, was wir vorfinden, ist unser nicht abzuschüttelndes Schicksal. Wir sind zum Anknüpfen an Gegebenes verurteilt. Eine der entscheidenden Vorgaben unseres Lebens sind wir selbst; wir haben uns nicht nur nicht die Familie ausgesucht, in der wir das Licht der Welt erblickt haben, wir haben uns auch selbst nicht ausgesucht. Uns bleibt nur, uns hinzunehmen. Wie, so könnte man einwenden, kann man dann aber von selbstbestimmtem Leben reden, wenn bereits die entscheidende Lebenserfolgsressource, wir selbst mit unseren Anlagen und Fertigkeiten, uns aufgezwungen worden ist. Ist Selbstbestimmung gebunden an absolute Freiheit und 
Voraussetzungslosigkeit, dann ist nur Gott zur Selbstbestimmung fähig, dann nur von ihm kann man sagen, dass er die Ursache seiner selbst ist und Raum und Zeit aus dem Nichts erschaffen hat.

Um die menschliche Freiheit vor der menschlichen Endlichkeit, vor den Notwendigkeiten der Welt und den Zufälligkeiten des Schicksals zu retten, hat die Philosophie zu einem alten Trick Zuflucht genommen, den sie immer anwendet, wenn sich das Offenbare ihren Ansprüchen verweigert. Es ist der Trick der Verdoppelung: früher wurde er vor allem in Gestalt der Weltverdoppelung benutzt; in der Moderne findet er vorwiegend in der Variante der Ich-Verdoppelung Verwendung. Hinter dem empirischen, genetisch, historisch und gesellschaftlich imprägnierten Selbst taucht ein anderes, den kontingenten Verstrickungen entrücktes Selbst auf, rein, unhistorisch. Und dieses fahle Ich wird nun mit der Bürde der Freiheitsbewahrung belastet; es wird zum Autor des Lebens erklärt, zum Subjekt der freiheitsbewahrenden Selbsterschaffung des Menschen in Raum und Zeit. Es ist evident, dass dieses Ich nach dem Bilde des abendländischen Gottes geschaffen wurde. So wie dieser sich bei der Erschaffung der Welt im Menschen ein Ebenbild erschuf, so bildet umgekehrt der Philosoph das subjektivitätstheoretische Freiheitszentrum nach dem Muster Gottes. Nichts weniger als ein selbstmächtiger Hervorbringer freier Handlungen, der keinerlei Voraussetzung bedürftig ist, nur sich selbst voraussetzen muss, nichts weniger als ein selbst unbewegter Beweger ist dieses Selbst.

Stärksten Ausdruck findet diese theomorphe Freiheitskonzeption in der Kantischen Theorie des autonomen Willens. Kants Autonomieverständnis hat nichts mit dem Allerweltsverständnis von Autonomie zu tun. Das Allerweltsverständnis von Autonomie orientiert sich am Begriff der Handlungsfreiheit. Autonom ist der, der keinem fremden Willen unterworfen ist, der innerhalb der koexistenzverbürgenden politischen Institutionen ein eigenbestimmtes Leben führen kann. Wer aber verbürgt, dass hinter diesen äußerlich unerzwungenen Handlungen auch ein freier Wille steht, dass wir nicht vom Man regiert werden? Handlungsfreiheit impliziert nicht Willensfreiheit; genauso wenig wie umgekehrt Willensfreiheit Handlungsfreiheit impliziert. Handlungsfreiheit existiert, wenn es keinerlei menschenverursachte äußere Handlungshindernisse, keinerlei menschenverursachte Handlungszwänge gibt. Willensfreiheit hingegen verlangt mehr: Willensfreiheit verlangt ? wie Kant es formuliert hat ? Kausalität aus Freiheit, verlangt, dass der Prozess der Absichts- und Willensbildung nicht heteronom verursacht ist, weder durch die Gesetze der inneren Natur noch durch anerzogene Überzeugungen und vorfindliche Üblichkeiten. Gibt es Kausalität aus Freiheit, dann gibt es für den Handelnden die Möglichkeit, sich von allen äusseren und inneren naturgesetzlichen Zusammenhängen und allen gesellschaftlich-heteronomen Bestimmungen gleichermassen zu distanzieren und zum einzigen Autor seiner Handlungen zu werden. Der freie Wille ist eine Freiheitsursache, die anders als alle anderen physikalischen und psychischen Ursachen nicht ihrerseits verursacht ist. Er hat keinerlei kausale Voraussetzungen und kann darum unvermittelt und voraussetzungslos, wie der welterschaffende Gott, wirksam werden. Jede freie Handlung hat darum den Charakter einer freien Schöpfung.

Diese gegen alle natürlichen und gesellschaftlichen Bestimmungen abgeschottete Enklave freier Selbsttätigkeit gibt es natürlich nicht. Es ist irrig, einen selbstverantwortlichen Personalitätskern aus der Hülle seiner natürlichen und sozialen Vorgegebenheiten herausschälen zu wollen. Es ist irrig, Willensfreiheit, Selbstbestimmung, Selbstverantwortlichkeit dadurch retten zu wollen, dass man ein mit erzeugungskausaler Unmittelbarkeit ausgestattetes hyperempirisches, supranaturales Subjektivitätszentrum installiert. Wenn wir an den selbstverständigungsrelevanten Prädikaten der Selbstbestimmung, Selbstverwirklichung, Ich-Identität und Ich-Authentizität nur dann festhalten können, wenn man einen cartesischen Geist in der Maschine unterstellt dann müssen wir lernen, ohne diese für uns so wichtigen Selbstzuschreibungen auszukommen. Dann müssen wir unser Freiheitskonzept halbieren und uns mit der Handlungsfreiheit bei evidenter Abwesenheit aller äusseren Zwänge begnügen.

Aber glücklicherweise müssen wir nicht zur schlechten Metaphysik der Ich-Verdoppelung Zuflucht nehmen, um unsere Freiheitsüberzeugung zu stützen, um den Begriffen der Selbstbestimmung und Selbstverwirklichung Sinn zu geben. Wir können auf die Kantische Radikalität verzichten und alle ontologischen Extravaganzen zurückweisen. Es ist nicht im mindesten erforderlich, den hypoleptischen Charakter menschlicher Existenz ontologisch zu unterlaufen und dem an die Hypolepsis gebundenen empirischen Menschen eine erfahrungsentrückte freiheitskausale Willensmitte einzureden.

Personen sind empirische Subjekte, mit unterschiedlichen und je unterschiedlich ausgeprägten Fähigkeiten und Kapazitäten ausgestattet, voller physischer, emotionaler, intellektueller und moralischer Bedürfnisse, zu einem Leben in vielfältigen praktischen Selbstverhältnissen, zur Selbstbeanspruchung und Selbstverantwortung, zur Selbstbestimmung, Selbstverwirklichung und Selbsterweiterung fähig. Personalität, Identität und Individualität moralischer Subjekte verweisen aufeinander und bilden eine Einheit, die ihre moralische Beurteilung zu würdigen und abzubilden hat. Personale Identität ist ohne körperliche Identität nicht möglich; körperliche Identität umfasst aber nicht nur die Bereitstellung eines Individualitätsprinzips, einer Verankerung der Person in Raum und Zeit. Körperliche Identität umfasst auch das gesamte Individualitätsmaterial, die gesamte empirische Beschaffenheit, die die Besonderheit, die Individualität der Person ausmacht. Eine personale Existenz besitzen, das Leben einer Person zu führen setzt also die Integration genau der empirischen Gegebenheit in die personale, in praktischen Selbstverhältnissen lebende Einheit voraus, von denen sich das Subjekt in der Kantischen Levitation ins Noumenale gerade zu entfernen trachtet.

Damit eine Person allerdings ein Leben führen kann und nicht lediglich von den Verhältnissen mitgenommen wird, muss sie sich Priorität gegenüber ihrer Zwecken, Wertvorstellungen und Verpflichtungen erarbeiten. Diese Prioritätsposition impliziert 
keinen cartesianischen Substantialismus, der das Selbst zu einer nicht-empirischen, geistigen Wirksamkeitssphäre machen, in eine immaterielle Substanz in einem materiellen Körper verwandeln möchte. Sie ist überaus ordinär, ein anthropologischer Gemeinplatz. Denn diese Prioritätsposition impliziert lediglich die unbestreitbar menschliche, gleichwohl in den einzelnen Individuen unterschiedlich ausgebildete Fähigkeit zur Reflexion. Diese Fähigkeit zur Reflexion ist mit der Tatsache durchaus vereinbar, dass wir sozial eingebettete und gesellschaftlich konstituierte Wesen sind, dass das menschliche Selbst immer ein ethisch parteiliches Selbst ist, das sich unabhängig von den identitätsbildenden Zugehörigkeiten nicht erkennen und losgelöst von den kollektiven Selbstverständigungsgrammatiken und kulturellen Interpretationskontexten überhaupt nicht verstehen kann. Unser höchstrangiges Interesse liegt darin, ein Leben zu führen, das wir für wertvoll erachten, das unseren Vorstellungen von einem guten Leben entspricht. Wir sind alle Glückssucher: wir richten unser Lebens im Netz der Besonderheiten ein. Diese Überzeugungen, die das Leben zu unserem machen und ihm den Charakter eines für uns sinnvollen Projekts verschaffen, sind jedoch ebenso wenig unhintergehbar wie der aus kollektiven Wertvorstellungen und Weltsichten gewebte Traditionshintergrund unserer gesellschaftlichen Lebensverhältnisse. Die Gesellschaft ist keine neutrale Bühne, auf der sich die Individuen wechselseitig bei ihrer Selbstfabrikation zusehen; sie prägt unsere Le-bensprojekte und wirkt bei unseren kommunikativ vermittelten Identitätserarbeitungen mit. Identitäten entstehen hypoleptisch, sie sind keine Vernunftgeburten, keine tabularasa-Konstruktionen, sondern Variationen vorgegebener Themen und Motive. Personen beziehen ihre Selbstverständigungsmaterialien aus einem immer schon kulturell bereitgestellten Vorrat an Beurteilungsperspektiven, Wertorientierungen und Solidaritätsmustern.

Wir besitzen jedoch die Fähigkeit, die selbst kulturell vermittelte und geschichtlich erworbene Fähigkeit, sowohl die kollektiven Überzeugungen des Traditionshintergrundes als auch unsere eigenen Vorstellungen von einem gelingenden Leben zu überprüfen, im Lichte neuer Erfahrungen neu zu bewerten, unter dem Eindruck neuer Argumente zu revidieren. Und diese Fähigkeit zur deliberativen Fortbildung unserer personalen Identität und unseres Lebensprojekts, zur reflexiven Distanzierung eingelebter Orientierungs- und Rechtfertigungsstandards ist uns wertvoll; sie ist konstitutiv für unser Verständnis von moralischer Subjektivität. Diese reflexions- und distanzierungsbegründete Freiheit liegt im Kern des modernen Freiheitsbegriffs und Autonomieverständnisses.

Dass diese Reflexions- und Distanzierungshandlungen ihrerseits wie alle menschlichen Handlungen voraussetzungsgebunden sind, die durch die Distanzierung möglich gewordene Überprüfung von Überzeugungen, Sichtweisen und Handlungsregeln sich ihrerseits voraussetzungsbeladener und in unüberprüfter Geltung stehender Standards und Kriterien bedient, ist kein Einwand gegen diesen reflexionsbegründeten Freiheits- und Selbstbestimmungsbegriff. Denn es ist keinesfalls notwendig, einen Zustand der Voraussetzungslosigkeit zu erreichen, um ein rational kontrolliertes Leben zu führen. Diese Vorstellung entstammt ursprungsmetaphysischen Vorstellungen, die sicheres Wissen von der kognitiven Verfügung über einen sich selbst ausweisenden Ursprung abhängig machen. Der nicht-abzuschüttelnde provisorische Charakter menschlicher Vernünftigkeit ist mit dem Bemühen um eine selbstbestimmte Lebensführung durchaus vereinbar. Menschliches Leben ist einem Floss vergleichbar, dem es verwehrt ist, in einem Trockendock überprüft zu werden. Wir können keinen menschenäußeren, lebensäußeren, kulturexternen Standpunkt beziehen, um mit universalen Standards unser Leben zu überprüfen. Wir können nicht unser Leben aussetzen, um uns ihm kritisch zuzuwenden. Wir können nur während unseres Lebens mit ihm ins Reine zu kommen versuchen. Verbesserungsarbeiten müssen während der Fahrt unternommen werden. Und dabei können wir nur mit den Materialien arbeiten, die wir mit unserem Leben selbst vorfinden.

Wenn von Freiheit und Selbstbestimmung in den Zusammenhängen der Moralphilosophie und der politischen Philosophie die Rede ist, dann wird genau dieses hermeneutische, nicht über den Rahmen unseres personalen Selbstverständnisses hinausreichende Freiheitskonzept zugrundegelegt. Über Freiheitsfähigkeit und Selbstbestimmungsfähigkeit verfügen Menschen, weil sie Vernunft und Selbstbewusstsein besitzen. Der Selbstbewusstseinsbesitz stattet sie mit der Fähigkeit zur Selbstbezüglichkeit aus. Personales Leben ist Leben in praktischen Selbstverhältnissen. Anderenfalls, wenn uns diese Selbstbezüglichkeitsfähigkeit verloren ginge, wussten wir nicht, wer wir sind, da wir Lebensgeschichte und Identität nur darum besitzen, weil wir als material sich zwar veränderndes, aber funktional sich über die Zeit hin kontinuierlich durchhaltendes Subjekt von Selbstzuschreibungen unterschiedliche Erfahrungen als eigene Erfahrungen haben, erinnern und zu einer biographischen Einheit zusammenbinden können. Ohne ein Subjekt, das diese Erfahrungen auf sich beziehen und sie daher zu längeren Episoden eigenen Lebens synthetisieren kann, gäbe es kein Leben, das wir gehabt haben. Und ohne Vernunft, die zum einen Erfahrungen gemacht hat, zum anderen Zukünftiges antizipiert und einen Lebensplan aus den vorfindlichen Möglichkeiten herauszupräparieren vermag, hätten wir keine Zukunft. Die Vernunft mischt sich, wie Kant es gesagt hat, in die „unmittelbar empfundenen Bedürfnisse” und stimmt ihre Befriedigung mit der „überlegten Erwartung des Künftigen ab” (Kant, 1902, S. 113). Zukunft ist die Fähigkeit, durch zukünftigen Hunger hungrig zu werden; Zukunft ist Erwartungsraum; durch seine antizipierende Öffnung vermögen wir überhaupt erst das Bestehende im Lichte von Alternativen zu sehen. Aufgrund ihrer grundlegenden Leistung der Transzendierung des Präsenten kreiert die Vernunft Optionen; damit legt sie den Grund zur Wahl und zur Freiheit der Wahl. Ohne diese vernünftige Zukunftserschließung wären wir am „Pflock der Gegenwart” (Nietzsche) gefesselt, handlungsunfähig, ausschliesslich auf gegebene Reize reagierend. Aber damit Vernunft und Selbstbezüglichkeit diese personalitätskonstitutiven Leistungen erbringen können, damit sie uns durch Identitätssicherung, Zukunftserschließung und Alternativenöffnung Freiheit und Selbstbestimmung ermöglichen, müssen sie nicht in einer der natürlichen und sozialen Kausalität entrückten cartesianisch-noumenalen Sphäre verankert sein.

\section{2. Über die Zumutungen der Freiheit, die immer grösser werden, je moderner die Gesellschaft wird}


Die Moderne macht angst, denn sie ist eine permanente Krise. Eine permanente Krise ist sie, da in ihr fortwährend, mit sich beschleunigendem Veränderungstempo, Gewohntes und Vertrautes ausser Geltung gesetzt wird. Damit geht der Orientierungsgewinn, den das Übliche abwirft, verloren. Wenn der Aufenthalt in der Gegenwart immer kürzer wird, die Gegenwart immer schneller veraltet und die Zukunft immer schneller eintritt, verlieren die Koordinaten, in denen wir unser Leben einrichten, die unsere Bewertungs- und Beurteilungsmassstäbe bestimmen, ihren Halt. Aber worin drückt sich der Krisencharakter der Moderne aus? Was ängstigt die Menschen so, bereitet ihnen so viel Ratlosigkeit und Unbehagen. Der Krisencharakter der Moderne ist in einem wachsenden Anstieg der Freiheit begründet. Wir leben in einer Freiheitswachstumsgesellschaft. Der Freiheitsanstieg hat zwei Wurzeln; da ist einmal der wissenschaftlich-technische Fortschritt; er vergrössert unsere Verfügungsmacht über die Natur, da ist zum anderen die schwindende Kraft der Institutionen; der Einfluss der Tradition, der herkömmlichen Lebensformen auf unsere eigene Lebensgestaltung wird zunehmend schwächer. Beides konfrontiert den Menschen mit Problemen; denn Freiheitszuwachs bedeutet wachsenden Entscheidungsbedarf; und dieser hinwiederum führt zu einer Vergrößerung der Verantwortungs- und Rechtfertigungslast, die abzutragen angesichts wachsender Ungewissheit die Kräfte der Menschen zu übersteigen droht. Manche haben die Befürchtung, dass die Freiheitsfähigkeit des Menschen der von ihm selbst erzeugten Freiheitswirklichkeit nicht mehr gewachsen ist.

Es ist alter Brauch, den Menschen mit den Tieren zu vergleichen; und wie stets gehen die Meinungen auch hier auseinander. Die einen beklagen den Menschen, ?denn?, so das immer wiederkehrende Argument, ?zu keinem der Tiere, die den Erdball bevölkern, sei die Natur so grausam gewesen wie zu ihm". Und wenn ihm einmal das Glück widerfahren würde, in ein Tier, und sei es selbst ein Schwein, verwandelt zu werden, was denen, die auf der Insel der Zauberin Circe landen, ja durchaus passieren kann, dann würde er um nichts in der Welt in die alte Gestalt und das elende menschliche Leben zurückkehren wollen. Das jedenfalls verkündete der einschlägig verwandelte Gryllos bei Plutarch in einem Dialog mit dem bezeichnenden Titel ?Die Vernunft der unvernünftigen Tiere? Odysseus, als dieser während seiner Reise auch die Insel der Zauberin besuchte und gern mit einigen zurückverwandelten Griechen ? die Tiere anderer Nationen interessierten ihn weniger ? nach Haus zurückgekehrt wäre.

Andere jedoch sehen das mit den Menschen und Tieren ganz anders; zum Beispiel Pico della Mirandola in seiner berühmten Rede „Über die Würde des Menschen” von 1486. Der Philosoph will hier den Nachweis erbringen, daß die vielbeklagte menschliche Mängelausstattung in Wirklichkeit ein unschätzbarer Vorzug sei. Keiner sei je reichhaltiger beschenkt worden als der, dem nichts gegeben wurde. Denn Gott, so heisst es dort, „sprach zu dem Menschen: Wir haben dir keinen bestimmten Wohnsitz, noch ein eigenes Gesicht, noch irgendeine besondere Gabe verliehen, damit du jeden beliebigen Wohnsitz, jedes beliebige Gesicht und alle Gaben, die du dir sicher wünschst, auch nach deinem Willen und nach deiner eigenen Meinung haben und besitzen mögest. Den übrigen Wesen ist ihre Natur durch die von uns vorgeschriebenen Gesetze bestimmt und wird dadurch in Schranken gehalten. Du bist durch keinerlei unüberwindliche Schranken gehemmt, sondern du sollst nach deinem eigenen freien Willen, in dessen Hand ich dein Geschick gelegt habe, sogar jene Natur dir selbst vorherbestimmen. Ich habe dich zur Mitte der Welt gemacht, damit du von dort bequem um dich schaust, was es alles in dieser Welt gibt" (Mirandola, 1984, S. 347).

Der Mensch, das ist die emphatische Botschaft dieser Rede, ist ein maßloses Wesen. Er besitzt eine uneingeschränkte Freiheit. Von allen natürlichen Festlegungen ungehindert vermag er über alle Natur zu verfügen, über die äussere so gut wie über die innere. Stets von neuem kann er sich nach seinem eigenen Bilde formen. Diese Rede ist ein beeindruckendes Dokument eines Epochenanfangs, des Anfangs der Moderne. Die lange dunkle Nacht des Mittelalters ist vorüber; der Morgen graut und selbstmächtig macht das Individuum sich daran, den Tag zu erobern.

Dort, in dem Gryllos-Dialog, also der Mensch in den Schöpfungsniederungen, erbärmlicher als jedes Tier, hier, in der Mirandola-Rede der Mensch als freie Vernunft in der Weltenmitte, allen Geschöpfen überlegen. Schaut mach jedoch etwas genauer hin, dann zeigt sich, dass diese beiden Positionen sich keineswegs grundsätzlich widersprechen: sie stehen zueinander wie die beiden Seiten einer Medaille: sie teilen die These von der wesentlichen Naturexternität des Menschen, geben dieser These aber eine unterschiedliche Interpretation: für den einen ist die naturrexterne Stellung des Menschen der Grund seiner Erbärmlichkeit, für den anderen ist sie der Grund seiner Exzellenz.

In der Tat nimmt der Mensch eine Sonderstellung unter den Lebewesen ein. Diese Sonderstellung hat zwei Gründe, die in den beiden erwähnten Texte auch anklingen. Wenn menschliche Selbstinterpretationen so weit auseinanderdriften, kann man sicher sein, dass sie beide wahr sind. Der von den Pessimisten hervorgehobene Grund der Sonderstellung liegt darin, dass der Mensch natürlich unterprivilegiert, weil unspezialisiert ist und keine ihm natürlich angepasste Umwelt besitzt, mit der er in natürlicher Harmonie lebt. Wir sind die Zukurzgekommenen der Schöpfung, unsere natürliche Ausrüstung ist selbsterhaltungs-mangelhaft, wir sind ökologisch obdachlos. Gäbe es da nicht den anderen, den optimistischen Grund der menschlichen Sonderstellung, wir wären längst nicht mehr. Dass wir noch sind, hat seinen Grund darin, dass wir, einerseits natürliche Heimatlose, Außenseiter, Habenichtse, doch andererseits in unserer kärglichen Naturausstattung Eigenschaften vorfinden, die als Verstand, Vernunft, Phantasie, Lernfähigkeit, Erfindungsreichtum, Anpassungsfähigkeit, Neugier bezeichnet werden, und die hier nur als Manifestationen der einen grundlegenden Fähigkeit interessieren, unsere natürlichen Mängel effektiv zu kompensieren. Das geschieht auf zwei Wegen: durch die Erschaffung von Werkzeugen und durch die Gewinnung von Wissen. Der Mensch ist ein Werkzeug erschaffendes Wesen, er hat aus biologischen Gründen Technik nötig. Damit er sich als Lebewesen in der Natur behaupten kann, muss er aus der Natur heraustreten und eine Werkzeugkultur ausbilden, die dann, da durch kein natürliches 
Mass gebunden, ihre eigene evolutionäre Dynamik entfaltet und den Naturgegebenheiten eine künstliche Welt menschlicher Erfindungen überstülpt.

Der Mensch verbessert seine Werkzeuge unaufhörlich; sein technologisches Verhältnis zur Natur ist in stetem Wandel begriffen. Dabei ist schon früh die Schwelle von der nur existenzsichernden und lebenserhaltenden Technik zur freiheitserweiternden und lebensoptimierenden Technik überschritten worden. Ging es ursprünglich darum, der Natur die Überlebensmittel abzuringen, so ging es im weiteren Fortgang der kulturellen Entwicklung darum, die Handlungsmöglichkeiten des Menschen zu vergrössern, den Zumutungen der Natur immer erfolgreicher zu begegnen und die Grenzen, die die Natur den Absichten und Wünschen der Menschen setzte, immer weiter hinauszuschieben. Durch die Technik emanzipiert sich der Mensch von der Natur, verringert er die Macht des Schicksals. Und die Ökonomie sorgt dafür, dass diese technikinduzierte Freiheitserfahrung nicht ein Luxusgut bleibt, sondern zu einem Massenprodukt wird. Diejenigen, die ihr antimodernistisches Unbehagen pflegen und auf die reißenden Veränderungen der wissenschaftlich-technischen Zivilisation mit einer Verteufelung der Technik reagieren, missachten das ungeheure gattungsgeschichtliche Emanzipationspotential, das in der technischen Entwicklung entfaltet wurde, begreifen nicht, dass mit der Wiederbelebung des Naturwüchsigen die kulturelle Regression beginnt, dass gerade in dem Unnatürlichen von Kultur und Technik menschliche Freiheit und menschliche Würde begründet sind.

Die zweite Quelle des modernitätstypischen Freiheitszuwachses ist der Autoritäts- und Geltungsschwund der sozialen Hierarchien, der kulturellen Regelsysteme und lebensweltlichen Institutionen. Das prominenteste Opfer der modernisierungsbedingten Erosion der lebensweltlichen Institutionen ist der Verbund aus Ehe und Familie. Der Wertewandel, das immer stärkere Individualisierungsbestreben hat auch Ehe und Familie nicht unverändert gelassen. Je mehr sich der Selbstverwirklichungsanspruch von den institutionellen, auf lebenslängliche Wirksamkeit ausgelegten Loyalitäten der Ehe emanzipiert, um so stärker büsst die Ehe an Geltung ein. Auch das sie schützende Recht konnte sich nicht gegen die Tendenzen der Zeit stellen und ist dem Wandel der Lebensorientierungen gefolgt. Der entscheidende Wendepunkt in der Entwicklung war die moralische Enttabuisierung der Scheidung. Sie führte in der Folgezeit zur Normalisie- rung der Scheidung und schließlich zur Aufnahme der Scheidung ins partnerschaftspolitische Alltagsinstrumentarium. Die Folgen dieser Entwicklung sind weitreichend. Die eheliche Partnerschaft steht unter steten Bewährungsdruck. Denn ist die Scheidung eine moralisch neutrale Normalbeendigung von Eheverhältnissen, dann ist die Aufrechterhaltung der Ehe zu jedem Zeitpunkt eine Wahlhandlung, die ihre Vorzugswürdigkeit gegenüber der Beendigung aufzeigen muss und damit unter Rechtfertigungszwang gerät. Die Fortgeltung eingelebter Traditionen verlangte wenig mehr als die Abwesenheit gravierender Missstände; erst recht war der Bestand der Ehe dann gesichert, wenn sie intern durch ökonomische Abhängigkeit des einen Partner vom anderen stabilisiert wurde. Verwandelt sich jedoch die Ehe aufgrund der zunehmenden ökonomischen Selbständigkeit des traditionell abhängigen Partners in ein symmetrisches Partnerschaftsverhältnis, das aufgelöst werden kann, ohne moralischen Tadel, gesellschaftliche Ächtung und kirchliche Sanktion befürchten zu müssen, dann muss die Partnerschaft zu jedem Zeitpunkt sich gegen die Alternative Scheidung behaupten können. Das treibt die partnerschaftsethischen Ansprüche an die Ehe, die Glückserwartungen der Partner an einander in die Höhe; eheliche Verbindungen sind darum in der Gegenwart mit einem beträchtlichen und stetig wachsenden Unzulänglichkeitsrisiko belastet; die Chance, das einer von beiden seine Gewinnerwartungen nicht mehr erfüllt sieht, sind gross. Eheliche Partnerschaften sind heute zerbrechliche Glücksversorgungsgemeinschaften von eigenem Wert, ohne institutionellen Außenhalt, ohne gesellschaftlichen Traditionsschutz. Sie müssen gepflegt und gehegt werden. Ihre Qualität ist allein abhängig von den emotionalen und ethischen Investitionen der Ehepartner. Kein Wunder, dass die Scheidungsraten rapide steigen; dass Beziehungsstress zu einer volksweiten Unbekömmlichkeitsquelle geworden ist. ?? Es ist nicht verwunderlich, dass diese dynamische Individualisierung der Ehe Auswirkungen auf die Familienstruktur hat. Wenn die maritale Axe ihren festen Halt verliert, gerät das ganze Gebilde ins Schlingern. Vielgesichtig ist heute die Familie. Unterschiedlichste Lebensführungsweisen vereinen sich unter dem weiten rechtlichen Dach der Familie ? schon lange ist die Lebensform des verheirateten Paares mit Kindern nicht mehr vorherrschend. Die moderne Familie leidet erheblich an personeller Auszehrung ? immer mehr Alleinerziehungsduale, immer mehr Kinderlose, die sich zu einer Steuersplittingkooperative zusammenfinden.

Die Verwandlung der technischen Verfügungsfreiheit und der ethischen Gestaltungsfreiheit in Selbstmächtigkeit ist die grundlegende Aufgabe, die die modernen Menschen bewältigen müssen. Um sie bewältigen zu können, müssen moderne Individuen die Fähigkeiten ausbilden, die es ihnen ermöglichen, die vorfindlichen Optionen zu ordnen, verantwortungsvoll zu gewichten und zu einem Lebensprogramm zu verdichten, in dessen Mittelpunkt die Selbstsorge steht. Denn in einer Gesellschaft der überbordenden Möglichkeiten und der schwindenden Bindungen rücken die Selbstmächtigkeit und Verantwortungsfähigkeit des Individuums ins Zentrum. Wir wollen natürlich gern Entlastung, wollen drückende Entscheidungen abschieben, auf die Politik, die Religion, die Moral; wir wollen, dass unsere Entscheidungsräume eingegrenzt werden, sind mit Entscheidungsfreiheit in kleinen, bekömmlichen Rationen zufrieden. Aber die Zeiten seliger Unmündigkeit sind endgültig vorüber. Autoritäten sind knapp geworden. In der Moderne schlägt die Stunde des Individuums.

\section{Die Selbstabschaffung des Bürgers im Sozialstaat}

Politik ist die dauerhafte Beendigung von Ausnahmezustand und Grenzsituation. Daher kann der Wechsel vom Naturzustand zum Staat auch als Übergang von der Notwendigkeit der Überlebenssicherung zur Möglichkeit der Lebensführung charakterisiert werden. Die politische Ordnung setzt die Herrschaft der Normalität durch; und Normalität herrscht dann, wenn durch die staatlichen Sicherheitsleistungen und die Festigkeit der Institutionen die basalen Voraussetzungen für menschliches 
Glück und Handlungserfolg, für existentielle Selbstentwicklung und ethische Selbstverwirklichung zur unauffälligen Selbstverständlichkeit geworden sind, wenn Gewalt aus dem zwischenmenschlichen Raum verbannt ist, Zukunftsvertrauen besteht, Erwartungen handlungsleitende Stabilität gewinnen und wechselseitige Verlässlichkeit herrscht. Aber nur dann findet diese staatliche Ordnung allseitige Zustimmung, wenn sie nicht der Durchsetzung besonderer moralischer, ethischer oder religiöser Vorstellungen dient, sondern ausschliesslich eine Ordnung der Handlungsfreiheit ist, die jedem einen gleich grossen Freiheitsraum zuteilt, in dem er in völliger ethischer und religiöser Unabhängigkeit sein Leben gestalten kann. Und dann ist diese Gesetzesordnung eine Ordnung der Freiheit, wenn die Gesetze ihre Legitimität von der Zustimmung der Bürger abhängig machen, wenn sie demokratischer Natur ist.

Betrachtet man diese liberale Ordnung aus der Perspektive der von ihr abgelösten Vergangenheit, dann erscheint sie als eine Ordnung des Negativen. Sie ist eine Ordnung des negativen Friedens, der negativen Freiheit, der negativen Gerechtigkeit. Negativen Frieden stiftet sie, da sie die Gewalt aus der Gesellschaft vertreibt; negative Freiheit ermöglicht sie, da sie alle Bindungen unter das Legitimationsmerkmal der Freiwilligkeit stellt; und negative Gerechtigkeit herrscht in ihr, insofern rechtsstaatlich garantiert wird, dass die Individuen in ihrer Willkür ausschliesslich durch formale und allgemeine Verbotsregeln eingeschränkt werden. Eine Ordnung des Negativen ist die liberale Ordnung also, weil sie eine Kollektivordnung der Abwesenheiten ist, der Abwesenheit von Gewalt, Zwang, herrschaftsrechtlicher Abhängigkeit und paternalistischer Bevormundung. Dass diese Negativität immer noch negativ beurteilt wird, als Makel und Mangel angesehen wird, beweist, dass der Liberalismus argumentationspolitisch und mentalitätspolitisch versagt hat: argumentationspolitisch, weil es ihm nicht gelungen ist, die Legende von der legitimationstheoretischen Konkurrenz negativer und positiver Freiheit zu zerstören und den Mythos von der positiven Freiheit als der legitimationstheoretisch überlegenen normativen Orientierung semantisch zu entlarven; mentalitätspolitisch, weil er es nicht geschafft hat, das Unbehagen an der Moderne umzudrehen und den Bürgern klar zu machen, welche Ausweitung an Lebensführungschancen sich hinter den modernitätsspezifischen Zumutungen der Individualisierung und Pluralisierung verbergen.

Die liberale Offenheit eigener Lebensgestaltung in einem Raum gewaltfreien Wettbewerbs behagt vielen nicht. Sie wollen nicht negativen Frieden und negative Gerechtigkeit; sie sind zumeist von einer pubertär-moralischen Sehnsucht nach ethischen Vollkommenheitsszenarien, religiösen Heilszuständen oder dem eudämonistischen geschichtsphilosophischen Finale erfüllt. Und in einem Leben auf solche utopische Horizonte hin soll dann die Existenz den begehrten Sinn, die Freiheit die wertvollere positive Färbung bekommen. Paradoxerweise wird damit Freiheitszerstörung als überlegene Freiheit ausgegeben; erst dann erweist sich der Mensch diesem Verständnis zufolge als eigentlich frei, wenn er sich den Imperativen erfüllten Menschseins unterwirft, die wohlgemerkt nicht in der Boutique der Postmoderne einschlägig Sinnbedürftigen ein harmloses existentielles Angebot machen, sondern sich als absolute Wahrheit verstehen, die sich nicht scheut, zu ihrer Durchsetzung auch auf Feuer und Schwert zurückzugreifen.

Für Wilhelm von Humboldt, Kant und den klassischen Liberalismus war mit der Errichtung einer rechtsstaatlich organisierten und demokratisch regierten Marktgesellschaft das Ziel der Politik erreicht. Allen Forderungen der Gerechtigkeit war in einer solchen Ordnung Genüge getan. Diejenigen, die mehr Gerechtigkeit wollten, als Rechtsstaat und Marktgesellschaft lieferten, als die Gleichheit vor dem Preis und die Gleichheit vor dem Recht garantieren konnte, durften sich nicht mehr an die Politik wenden; sie mussten zur Religion ihre Zuflucht nehmen und auf die Kompensationsleistungen postmortaler Sanktions- und Gratifikationssysteme hoffen.

Jedoch bei dieser Arbeitsteilung zwischen irdischer Freiheitsordnung und jenseitiger Heilserfüllung ist es nicht geblieben. Der Anspruch an die institutionellen Rahmenbedingungen individueller Lebensplanung ist in der individualistischen Moderne unaufhörlich gestiegen. Der Bereich der politischen Verantwortlichkeit weitete sich stetig. Der Rechts- und Verfassungsstaat wandelte sich zum Sozialstaat, der tief in die wirtschaftlichen Abläufe und gesellschaftlichen Beziehungen der Menschen eingriff. Die Verantwortlichkeiten der Bürger wurden ausgedünnt, die Zuständigkeiten des Staates hingegen wuchsen weiterhin. Kämpfte er ursprünglich an den Grenzen der Normalität gegen den eindringenden Naturzustand, so wurde er im Gewand des Sozialstaats, unter den Bedingungen demokratischer Herrschaft, allgegenwärtig, aufdringlich. Eine stetig wachsende Bürokratie der Beobachtung, Betreuung und vorauseilenden Sorge entstand. Anfänglich ging es nur um die Domestikation des Leviathans, um den Schutz der Bürger vor dem Golem, den sie selbst geschaffen hatten, um sich voreinander zu schützen. Durch institutionelle Fesseln unterschiedlichster Art wurde seine Bewegungsfreiheit eingeschränkt. Seine wilde Natur wurde durch die Moral der Menschenrechte besänftigt; und sein entschlossen zupackendes, entscheidungsschnelles Wesen durch die mühselige Konsensfindungsmaschinerie der demokratischen Organisationen gelähmt. Doch mit Einrichtung und Ausbau des Sozialstaats endete dieser Prozess der Selbstermächtigung der Bürger. Es kam zu einer Erneuerung des leviathanischen Tauschgeschäfts. Jetzt jedoch wurde nicht Lebensschutz gegen Rechtsgehorsam getauscht, sondern Loyalität mit der Sicherung dynamisierter Lebensqualität vergolten. So wurde es zur bürgerlichen Gewohnheit, den Staat für alle Lebensumstände verantwortlich zu machen, ihn, wie früher die Götter, als Schutz gegen alle Widrigkeiten des Schicksals anzurufen. Und er hat, anders als die Unsterblichen, den Gläubigen stets Gehör geschenkt.

Liberale Ordnungspolitik wird so den machtpolitischen Verheißungen einer benevolenten massendemokratischen Wählerbewirtschaftung geopfert. Die zur dürftigen moralischen Tarnung gern aufgegriffene Totalverantwortlichkeitsunterstellung stützt sich auf ein begrifflich diffuses und normativ vages sozialstaatliches Legitimationskonzept, das Maßlosigkeit begünstigt und allen Begehrlichkeiten der organisierten Interessengruppen moralische 
Rückendeckung verspricht. Sozialstaatliche Staatszweckbestimmung schwankt zwischen den Zielen der Daseinsfürsorge und der Verteilungsgerechtigkeit, zwischen kompensatorischer Ungleichheitsminderung und Chancengleichheitsgewährleistung, zwischen bedürfnisorientierter Grundversorgung, exklusionsverhindernder bürgerlicher Solidarität, flächendeckender Diskriminierungsabwehr und kulturellem Identitätsschutz. Und so unübersichtlich der Begriff, so unübersichtlich auch die bürokratische Wirklichkeit. Die Ordnungen der Sicherheit und Freiheit drohen unter dem Druck überbordender Verantwortlichkeit und Zuständigkeit zu zerbrechen. Im Gestrüpp der wuchernden leistungsstaatlichen Bürokratie blüht Misswirtschaft, greifen Zerfall und Korruption um sich. An der Komplexität der Institutionen der sozialstaatlichen Eingriffsverwaltung verschleißt sich die Gestaltungskraft der politischen Intelligenz. Jede Problemlösung erzeugt aufgrund der Unübersichtlichkeit der Verhältnisse und der kognitiv undurchdringlichen Regulationsdichte der rechtlichen Regelungen nicht vorhersehbare neue Probleme. Die sozialstaatliche Politik wird unordentlich, das Gemeinwesen wird unregierbar. Und nach wie vor wird diese horizontlose Flickschusterei begleitet von einem vollmundigen Moralismus, von einem Sekuritäts- und Gerechtigkeitspopulismus, der weder die ökonomischen noch die moralischen Folgen seines partei- und verbandspolitischen Egoismus bedenkt. Dem Ziel der „Vergerechtlichung” (Bernard Schlink) aller Lebensverhältnisse verschrieben ist der Staat immer weniger imstande, seiner fundamentalen Verantwortlichkeit, nämlich der Ordnungsvorsorge, gerecht zu werden, stabile Rahmenbedingungen des gesellschaftlichen Lebens zu garantieren, in denen die Bürger ein Leben mit Eigenbeteiligung führen und ein selbstverantwortliches Risikomanagement entwickeln können.

Natürlich wird auch eine von dieser Allzuständigkeitsanmassung befreite Sicherheits- und Freiheitsordnung sozialstaatliche Aufgaben wahrzunehmen haben. Diese dienen jedoch nicht einer chimärischen Verteilungsgerechtigkeit, sondern der Bereitstellung einer Grundversorgung für Selbstversorgungsunfähige und der Errichtung eines institutionellen Rahmenwerks zur Gewährleistung gleicher Lebenschancen. Was aber heisst Chancengleichheit? Es ist hilfreich, hier zwei Lesarten von Chancengleichheit zu unterschieden. Da ist einmal die Gerechtigkeit der flachen Chancengleichheit und da ist zum anderen die Gerechtigkeit der tiefen Chancengleichheit. Während die flache Chancengerechtigkeit auf die Etablierung eines rechtsstaatlichen Rahmens individueller Lebensführung zielt, um einen diskriminierungsfreien Wettbewerb um Positionen, Ämter und Arbeitsstellen zu gewährleisten, geht es der tiefen Chancengleichheit um umfassende Gleichheit der Startbedingungen. Flache Chancengleichheit erhebt keinen Einspruch, wenn die vorgefundene, in jeder Hinsicht zufällige, durch keinerlei individuelle Leistung verdiente genetisch-soziale Prägung den Bewerbungserfolg der Individuen bestimmt, wenn sich die Ungleichheit der genetisch-sozialen Ausgangslage in soziale und ökonomische Ungleichheit verwandelt. Flache Chancengleichheit erhebt erst dann einen Einspruch, wenn die selektiven Effekte diskriminierender Regeln, Praktiken, Sichtweisen einen offenen Wettbewerb der Individuen um Ämter, Positionen und Arbeitsstellen verhindern. Diese Ungleichheitstoleranz hingegen vermag der Anhänger der tiefen Chancengleichheit nicht aufzubringen. Denn natürlich ist die Qualität der Lebenskarrieren der Individuen abhängig von ihren natürlichen und erworbenen Fähigkeiten und Fertigkeit. Die Talentierteren und durch günstige Sozialisationsbedingungen Geförderten können mit einer weit besseren Ressourcenausstattung ins Leben gehen als ihre genetisch weniger gut bedachten und sozial weniger begünstigten Mitbewerber. Und das ist in den Augen des Egalitaristen ein gerechtigkeitsethischer Skandal.

Wie weit mag der Egalitarismus der Lebenserfolgsressource, die wir selbst sind, gehen? Schönheit, zumal in einer so äußerlichkeitskultischen Gesellschaft wie der unsrigen, ist eine soziale Macht. Muss nicht angesichts der überaus kläglichen Ergebnisse der natürlichen Ästhetiklotterie einerseits und der unleugbaren Startvorteile der Schönen andererseits der Egalitarist revoltieren? Eine Schönheitssteuer einführen oder freie Kosmetik oder freies Hanteltraining für alle einschlägig Bedürftigen? 1960 hat L. P. Harley in London ein Buch mit dem schönen ?Titel Facial Justice? veröffentlicht. Es berichtet von dem Gerechtigkeitsskandal der Schönheit, von dem unverdienten guten Aussehen, dem Wettbewerbsvorteil der angenehm geschnittenen Zügen, von der benachteiligenden Hässlichkeit und der marginalisierenden Unansehnlichkeit. Und es berichtet von der „Antlitz-Gleichmachungs-Behörde” und ihrem Egalisierungsprogramm, das durch die Entwicklung einer risikolosen und unaufwendigen Gesichtschirurgie ermöglicht wurde und erlaubte, die blinde natürliche Verteilung ästhetischer Eigenschaften durch Gesichtsplastiken der ausgleichenden Gerechtigkeit zu überformen, so dass nur noch ästhetische Durchschnittlichkeitsvarianten existierten und die körperliche Individualität sich auf eine karrierepolitisch neutrale Mediokritätsvariation beschränkte. Damit hätte die „Antlitz-Gleichmachungs-Behörde” dann die Ungerechtigkeit der Natur überwunden und die soziale Macht der Schönheit gebrochen. Der Egalitarist, seit je Anwalt neidischer Vergleichssucht, kann zufrieden sein: die Schönen sind verschwunden und die ästhetischen Habenichtse müssen sich nicht mehr in Neid zer-fressen.

Es ist ersichtlich, dass der Sozialstaat durch die Ausdehnung des Prinzips der Chancengleichheit auf den Bereich der natürlichen und sozialen Prägung, also durch eine Politik der tiefen Chancengleichheit, sich in eine totalitäre Bürokratie verwandeln muss. Die legitime Zuständigkeit staatlichen Eingriffshandelns endet an der Haut der Menschen. Daher ist die genetische und soziale Konditionierung menschlichen Lebens kein legitimer Gegenstand einer umverteilenden Gerechtigkeit. Wir sind Personen, die ein selbstverantwortliches Leben zu führen das Recht haben; und der Staat ist als Institution der Institutionen mit der Aufgabe betraut, ein System der institutionellen Sicherung einer ? notwendig flachen ? Chancengleichheit zu etablieren. Wir sind jedoch keine Lebenserfolgsressourcen, die durch Sozialstaatshandeln egalisiert werden müssen, um Chancenvorteile und Chancennachteile auszugleichen. Entsprechend ist auch die Ungleichheit als gerechtigkeitsethisch unbedenklich zu akzeptieren, die im Rahmen eines Systems der flachen Chancengleichheit durch die unterschiedlichen genetischen und sozialen Prägungen produziert wird. 
Wenn sich staatliches Handelns als individuensensibler Schicksalsausgleich versteht, wird die Dimension des Politischen zerstört. Politik ist nicht mehr Sorge um den Bürger, nicht mehr Diskriminierungsbekämpfung und Sorge um Bürgerlichkeit ermöglichende Umstände. Der Bürger ist in diesem egalitären Gerechtigkeitsstaat längst ausgestorben. In der Welt des Egalitarismus gibt es nur Bevorzugte und Benachteiligte, Schicksalsbegünstigte und Schicksalsbeladene, sozial Privilegierte und sozial Deprivilegierte. Und jeder Benachteiligte hat die Definitionshoheit über seine Benachteiligung und das kompensationspflichtige Ausgleichsausmaß, ist zur eigenständigen Handhabung des Lebenskarriereabstandsmessers befugt. Damit ruft diese egalisierungsverpflichtete Gesellschaft zur Selbstorganisation des Neides auf. Eine paradoxe Tiefenstruktur des Egalisierungsetatismus wird sichtbar: der Egalitarismus produziert nicht nur Gleichheit, sondern notwendig immer auch ihr Gegenteil; als Inegalitätskompensation schüttet er fortwährend Prämien für Inegalität aus und erklärt damit die Autoviktimisierung zur Erfolgsstrategie.

Auf merkwürdige Weise wiederholt sich in der Gesellschaftsauffassung des Egalitarismus damit die Hobbes?sche Naturzustandslogik. Beide Gesellschaften sind Komparativgesellschaften, Gesellschaften des Vergleichs, der Kultivierung des Abstands. Zielt alles unter Naturzustandsbedingungen auf Machtvorsprung, so zielt alles in dem Gleichheitsstaat auf Benachteiligungsvorsprung. Die Egalitaristen ersetzen den positiven Komparativ durch den negativen Komparativ. Das hat politisch und moralisch verhängnisvolle Konsequenzen. Die Autoviktimisierung verlangt nach einer Dramatisierung der Ungleichheit. Ungleichheitsdramatisierung verträgt sich aber nicht mit einer differenzierten Ungleichheitsheitwahrnehmung. Die Ungleichheit der Ungleichheiten gerät aus dem Blick, differenzierungsunfähig ebnet der Egalitarist alle Ungleichheitsunterschiede ein. Daher ist der Dramatisierung von Ungleichheit immer die Gefahr der Verharmlosung von Armut, Not und Elend, von Unterdrückung, Ausbeutung und Erniedrigung eingeschrieben. Denn entweder wird der Egalitarist den moralpolitischen Gewinn realisieren wollen, der mit der Verschleifung der internen Ungleichheitsstufen verbunden ist und jede Gleichheitsabweichung, jede vergleichsweise geringere Ressourcenausstattung, jede Benachteiligung mit den moralischen Schreckensfarben der Not und des Elends anmalen, oder er wird Armut, Not und Elend, Unterdrückung, Ausbeutung und Erniedrigung als Fall von Gleichheitsabweichung und als Folge geringerer Ressourcenausstattung verharmlosen und damit politische Ursächlichkeit und Verantwortlichkeit verwischen. In beiden Fällen riskiert das egalitaristische Programm moralische Glaubwürdigkeit und politische Ernsthaftigkeit.

Die Kehrseite der Autoviktimisierung ist die Stigmatisierung. Um besonders grosse Aus-schüttungen aus dem egalitaristischen Kompensationsfond zu erhalten, muss man besonders gravierende Benachteiligungen vorweisen. Und um seinen Benachteiligungsvorstellungen politische Anerkennung zu verschaffen, schliesst man sich am besten mit anderen zu Verbänden zusammen. Wenn wir aber nun nicht die karge Lebensethik eines Nutzenmaximierers zugrundelegen, sondern von einem anspruchsvolleren moralischen Subjekt ausgehen, das Respekt und Anerkennung verlangt, dann bietet das Leben in einer egalitaristischen Gesellschaft der Taxation des Benachteiligungs? und Bevorzugungsabstands nicht nur ungeahnte Möglichkeiten leistungsfreier Einkommensmehrung, sondern dann kann der Egalitarismus auch als Quelle der Stigmatisierung erfahren werden. Denn der ethische Preis für den Erhaltung der Benachteiligungsprämie ist Inferiorisierung und Erniedrigung. Der Egalitarismus macht ausschließlich das binäre Benachteiligungs?Bevorzugungs?Schema zur epistemologischen Grundlage seiner Gleichheitspolitik. Die von ihm bereitgestellten Transfereinkommen fließen von den Mehr?Menschen zu den Minder?Menschen, von den Begabteren, Leistungsstärkeren, Talentierteren zu den Minderbegabten, Mindertalentierten, Minderleistern. Der egalitäre Sozialstaat ist die institutionalisierte Herablassung. Aber diejenigen, die dagegen ethisch aufbegehren, sind gering an der Zahl.

Denn der Wohlfahrtsstaat hat die zurückliegenden Dekaden gut genutzt und sein eigenes kulturelles Binnenklima erzeugt. Er ist den Bürgern unter die Haut gegangen, hat ihr Denken, Handeln und Fühlen geprägt. Er ist ein Seelenbildner, der sich den Menschen nach seinem Bilde erschaffen hat. Vor dem Hintergrund der liberalen Freiheitsethik ist dem expansiven Wohlfahrtsstaat der Gegenwart entschieden der Vorwurf der moralischen Kontraproduktivität zu machen: er betreibt zügig die Abschaffung der Selbständigkeit, er verhindert Bürgerlichkeit. Während der Markt ein System der wechselseitigen Verstärkung ökonomischer und selbstverantwortungsethischer Anreizstrukturen bietet, eigenverantwortliche Lebensführung und ökonomische Erfolgssuche strukturell harmonisiert, treten verantwortungsethisches und ökonomisches Anreizsystem im Wohlfahrtsstaat in ein polemisches Verhältnis. In demselben Masse, in dem im solidaritätsbegründeten Wohlfahrtsstaat die Berechtigten zu Klienten werden und ökonomisch orientiertes Verhalten an den Tag legen, möglichst grosse private Ausnutzungsmargen suchen und sich politisch organisieren, um ihre gruppenbezogene Gesamtzuteilung zu erhöhen, verkümmern die verantwortungsethischen Anreize, die Selbstbeanspruchungsbereitschaft und das pure, nach Unabhängigkeit von fremden Erhaltungsleistungen trachtende Selbstständigkeitsbedürfnis. Die Bürger haben längst die staatliche Sicht der Dinge unternommen. Ihre existentielle Grammatik ist durch und durch welfaristisch konditioniert. Der Gewährleistungsbereitschaft des Staates korrespondiert eine umfassende bürgerliche Schutzforderung. Diese Selbstklintelisierung des Bürgers löst diesen aus dem Horizontalgeflecht der Gesellschaft, aus ihren Lernprozessen und Anpassungszwängen und stellt ihn in eine obrigkeitliche Vertikale. So wie im Protestantismus jedes Individuum unmittelbar zu Gott steht, so steht der Sozialstaatsklient zum Staat. Diese dekontextualisierende Position erklärt vermutlich auch, warum der Protestantismus solche Schwierigkeiten hat, die kapitalistische Wirtschaftsgesellschaft anzuerkennen, und ein so intimes, von keinerlei Bürgerskepsis getrübtes Verhältnis zum Sozialstaat entwickelt hat, wohingegen dem auf das Subsidiaritätsprinzip eingeschworenen Katholizismus diese Einstellung komplementärer Kapitalismusverteufelung und Welfarismusdivinisierung eher fremd ist. 
Liberale dürfen sich von der alles umspülenden Gerechtigkeitsrhetorik der Parteien, Verbände, Interessengruppen und Kirchen nicht beeindrucken lassen. Sie müssen den Mut aufbringen, ihrem großen Theoretiker Hayek zu folgen und sich zu einer öffentlichen Bekämpfung des Gerechtigkeitsbegriffs aufraffen. Als Füllhorn der Verteilungsgerechtigkeit entbehrt der Sozialstaat aller Legitimität. Er ist nur als freiheitsdienliche Veranstaltung grundrechtlich legitimierbar, als freiheitsdienliche Veranstaltung, die für formale Chancengleichheit sorgt, eine Grundversorgung der Selbstversorgungsunfähigen sichert, Marktpflege betreibt und mit effektivem marktwirtschaftlichen Instrumentarium zur Selbständigkeit ermutigt und individuelle Autonomie fördert. Der Sozialstaat muss auf die machtpolitischen Gewinne der Versorgungs- und Betreuungsstrategien verzichten, das Linsengericht-Agreement, in dem Freiheit gegen Versorgung, gegen Stallfütterung eingetauscht wurde, rückgängig machen und den Bürgern ihr freiheitsrechtliches Erstgeburtsrecht zurückgeben.

\section{Anhang}

Liberale, die immer schon dem Verteilungsparadigma des sozialdemokratischen Zeitalters misstraut haben, treten im gerechtigkeitstheoretischen Diskurs für das Konzept der flachen Chancengleichheit ein. Das ihm eingeschriebene Ideal ist eine Gesellschaft, in der alle gleiche Chancen haben, ein ihren Talenten, Begabungen und frei entwickelten Interessen entsprechendes Leben zu führen. Nun gibt es ethisch-religiöse Lebensentwürfen, die an gesellschaftlicher Teilhabe nicht interessiert sind, die geradezu den Kontakt mit der gesellschaftlichen Wirklichkeit meiden, weil sie die Einflüsse einer säkularen individualistischen Gesellschaft fürchten. Muss nicht eine Politik der Chancengleichheit diesen Lebensentwürfen genau dasselbe Recht auf Verwirklichung zubilligen, das sie den Lebensentwürfen einräumt, die aus der Mitte der individualistischen Gesellschaft stammen und deren individualistsches Ethos spiegeln? Schliesslich kann eine moderne Gesellschaft ihre Rechtsund Chancenzuteilung nicht an ethisch-religiöse Präferenzen binden.

Wo die Politik der Chancengleichheit entdifferenzieren will, vorhandene benachteiligungswirksame Unterschiede ausgleichen will, will der Multikulturalismus Differenzen geachtet und gesichert sehen; daher durchzieht ihn auch ein tiefer Argwohn gegenüber allen integrationspolitischen Bemühungen. Der Multikulturalist strebt kulturelle Selbsterhaltung an, verfolgt das Idealziel einer vollständigen Gleichstellung mit der Mehrheitskultur. Ihm geht es um eine Beendigung der politischen und strukturellen Privilegierung der individualistischen, säkularen, liberalen Lebensform der Stammkultur durch die Zuerkennung von Gruppenrechten und die Auflage kompensatorischer Programme, die allesamt dazu dienen, der kulturellen

Marginalisierung in einem durch liberale Indifferenzpolitik geprägten Gemeinwesen entgegenzutreten, und sei dies auch um den Preis der Verletzung der mehrheitskulturell essentiellen Prinzipien der Gleichheit und Neutralität. Der differenzpolitische Grundgedanke ist es, durch geeignete Zuweisung von Sonderrechtsmacht soviel politische Vertretungsmacht und Sozialmacht zu erhalten, dass die häufig einer älteren sozialgeschichtlichen Entwicklungsstufe angehörenden ethnisch-religiöse Gruppe in einer feindlichen kulturellen Umwelt überleben kann.

Aber hier ist an die menschenrechtliche Wurzel des Konzepts der Chancengleichheit zu erinnern. Menschenrechtswidrige Behandlungsweisen erfahren auch dann nicht durch ein Recht auf gleiche Lebenschancen gerechtigkeitsethische Rückendeckung, wenn sie Bestandteil identitätsbildender kultureller Praktiken und religiöser Überzeugungssysteme sind. Zwar gehört Neutralität gegenüber den unterschiedlichen individuellen und kollektiven Konzeptionen eines guten Lebens zu den vornehmsten Grundsätzen der modernen liberalen gesellschaftlichen Ordnung. Da aber dieses Neutralitätsprinzip seinerseits Ausdruck des menschenrechtlichen Egalitarismus, des gleichen Rechts eines jeden ist, sein Leben innerhalb der Gesetze des demokratischen Gemeinwesens selbst zu bestimmen, schließt sittlich-ethische Neutralität keinesfalls eine indifferente Haltung gegenüber menschenrechtswidrigen kulturellen Formationen ein. Die integrationspolitischen Herausforderungen an den Liberalismus wachsen, und sie wachsen um so mehr, je moderner die Moderne wird, je mehr die kulturelle Homogenität unter dem Pluralismusdruck verschwindet. In einer multiethnischen Gesellschaft der vielen Kulturen, Religionen und Geschichten fallen alle Instanzen aus, die traditionellerweise die Last der sozialen Kohärenz und der politischen Integration getragen haben. Weder kann man sich auf die Gemeinsamkeit der Nation noch auf die Gemeinsamkeit der Geschichte berufen. Denn die Migrationsgesellschaften der Gegenwart und Zukunft vereinigen Gruppen mit je eigenen Geschichten. Sie haben darum keine gemeinsame Vergangenheit, folglich auch keine geteilte politische Mythologie und Symbolik. Bestenfalls haben Migrationsgesellschaften eine gemeinsame Zukunft. Damit sie diese aber haben können, bedürfen sie eines Gemeinsamkeitsfundaments, das nur aus den Materialien des menschenrechtlichen Universalismus gebaut werden kann. Dieses Gemeinsamkeitsfundament muss verteidigt werden, nach innen wie nach aussen. Und dazu gehört eine Politik der Chancengleichheit, die sich durch identitätspolitische Einreden nicht beirren belässt.

Grenzenlose Toleranz ist Ausdruck ethischer Charakterlosigkeit und eines überzeugungsleeren Relativismus, ist wie grenzenlose Freiheit ein aporetisches, selbstzerstörerisches Konzept. Das Vernünftige kann nicht das Unvernünftige tolerieren. Der Demokrat kann nicht den Demokratieverächter tolerieren. Der Anhänger des menschenrechtlichen Egalitarismus kann nicht die menschenrechtswidrigen Praktiken fremder Kulturen und Religionen tolerieren. Fundamentalismus ist Menschenfeindschaft. Der Multikulturalismus ist eine Ausgeburt der Schwäche, des Verrats an den eigenen Überzeugungen. Nicht die Gruppe, sondern das Individuum ist der Protagonist und damit auch der Schutzbefohlene menschenrechtlicher Ordnungen. Würden die Helden der bürgerlichen Emanzipation sehen können, wie ihre Überzeugungen gegenwärtig differenzpolitisch und gruppenrechtstheoretisch verzerrt werden, wie ihr Liberalismus der Individuen in einen Kommunitarismus des Gruppenschutzes verkehrt wird, müssten sie mit ansehen, wie feige die meisten Wortführer des räsonierenden Publikums des Westens auf die Einschüchterungskulisse des eingeschleusten Fundamentalismus reagieren, wie die Intellektuellen erst vor den 
Dreistigkeiten zurückweichen und bei mörderischer Gewalt sofort nach Provokateuren im eigenen Lager Ausschau halten, sie würden sich ihrer Nachfahren schämen. Beurteilen wir die Überlebensfähigkeit der Freiheit nach dem Anblick, den die Tribune des Gutmenschentums seit geraumer Zeit bieten, muss jedem Freiheitsfreund bang ums Herz werden. Diese bislang so erfolgreiche, selbst den totalitären Bestien des Faschismus und Kommunismus trotzende geistige Formation muss sich dringend an das Einmaleins der ethischen Selbstbehauptung erinnern, muss wehrhaft werden.

Alle weltanschaulichen und religiösen Überzeugungssysteme, die die modernitätstypische laizistische Scheidung zwischen dem Öffentlichen und dem Privaten nicht kennen, haben ihr liberales Damaskus noch vor sich, und so lange sie das fällige Ausdifferenzierungspensum des Sittlich-Religiös-Kompakten in Recht, öffentlich-demokratische Diskursmoral und private Agathologie noch nicht hinter sich gebracht haben, stellen sie innerhalb der modernen Gesellschaft ein Konfliktpotential dar, das diskursiv und argumentativ nicht entschärft werden kann. Wenn die Konflikte zwischen den vernünftigen Überzeugungen und der fundamentalistischen Unvernunft den Bestand der Bürgerkultur gefährden, die Demokratie zerstören und die politische Gerechtigkeitsordnung umstürzen, dann erlischt die Toleranzpflicht. Wenn unvernünftige Lehren Einfluss auf die Politik nehmen und die staatliche Macht benutzen wollen, um ihre undemokratischen, menschenrechtswidrigen und illiberalen Vorstellungen zu verwirklichen, dann muss die Ordnung des politischen Liberalismus sich verteidigen. Und sie tut dies am besten, indem sie die unvernünftigen Lehren von Anfang an misstrauisch beobachtet und ihren Einfluss eindämmt.

Unvernünftige Lehren muss man, darin ist dem Egalitaristen Rawls einmal zuzustimmen, behandeln wie „Krankheiten und Kriege”. Man muss der epidemischen Ausbreitung der Unvernunft mit geeigneten Maßnahmen begegnen. Verfassungsfeindschaft lässt sich nicht mit Dialogangeboten, mit pädagogisierenden Strategien entschärfen; Überzeugungsarbeit ist erst dann zu leisten, wenn das zur Selbsterhaltung Erforderliche getan worden ist.

\section{Literatur:}

Kant, I. (1902). Muthmaßlicher Anfang der Menschengeschichte. In: Gesammelte Schriften. Akademie-Ausgabe, Bd. VIII, Berlin.

Mirandola, Pico della (1984). Über die Würde des Menschen. In: St. Otto (Hrsg.), Geschichte der Philosophie in Text und Darstellung: Renaissance und frühe Neuzeit. Stuttgart: Reclam.

Nietzsche, F. (1967a). Morgenröthe. Kritische Gesamtausgabe (= KGA). Bd. 5/1, hg. von G. Colli und M. Montenari, Berlin: de Gruyter.

Nietzsche, F. (1967b). Menschliches, Allzumenschliches. KGA, Bd. 4/3.

Nietzsche, F. (1967c). Zur Genealogie der Moral. KGA, Bd. 6/2.

Nietzsche, F. (1980). Menschliches, Allzumenschliches. In: F. Nietzsche, Werke in sechs Bänden, hg. von Karl Schlechta, Bd. 2. München: Hanser Verlag. 